\title{
Estudo epidemiológico de lesões odontogênicas provenientes do Departamento de Patologia e Medicina Legal da Universidade Federal do Amazonas
}

\author{
Epidemiological study of odontogenic lesions from the Department of Pathology and Legal \\ Medicine of the Federal University of Amazonas
}

\begin{abstract}
Brendo Vinicius Rodrigues Louredo, ${ }^{1}$ Camila Tatyanne Santos de Freitas, ${ }^{2}$ Jeconias Câmara, ${ }^{2}$ Tatiana Nayara Libório-Kimura ${ }^{2,3}$
${ }^{1}$ Programa Institucional de Bolsistas de Iniciação Científica (FAPEAM), Curso de Odontologia, Universidade Federal do Amazonas, Manaus, AM, Brasil

${ }^{2}$ Programa de Residência em Patologia Oral e Maxilofacial, Faculdade de Medicina, Universidade Federal do Amazonas, Manaus, AM, Brasil

${ }^{3}$ Programa de Pós-Graduação em Odontologia, Curso de Odontologia, Universidade Federal do Amazonas, Manaus, AM, Brasil

- Os autores declaram que não há conflito de interesse.
\end{abstract}

\section{Resumo}

Objetivo: estabelecer o perfil epidemiológico das lesões odontogênicas provenientes do Departamento de Patologia e Medicina Legal (DPML) da Faculdade de Medicina (FM) da Universidade Federal do Amazonas (UFAM), analisando os tipos histológicos mais prevalentes de cistos e tumores odontogênicos assim como suas características clinicopatológicas. Material e Métodos: cento e quarenta e oito casos de lesões odontogênicas, entre os anos de 1999 e 2014 , foram recuperados do DPML/FM/UFAM e analisados quanto ao sexo, idade, raça, localização anatômica e tipo histológico. Esses dados foram submetidos à análise descritiva. Resultados: do total de 148 pacientes acometidos por lesões odontogênicas, encontrou-se leve predileção pelo sexo masculino ( $54 \%, n=80)$, na segunda década de vida $(34,1 \%, n=47)$ e sendo a mandíbula o sítio anatômico mais acometido com $59,7 \%$ ( $n=77)$ dos casos. Quanto aos tipos histológicos das lesões, $68,9 \%$ ( $n=$ 102) foram de cistos e $31,1 \%(n=46)$ de tumores odontogênicos. Dos cistos, o tipo histológico mais prevalente foi o periapical $(39,3 \%, n=40)$ e dentre os tumores, o tipo mais comum foi o ameloblastoma $(45,6 \%, n=21)$. Conclusão: as lesões odontogênicas prevaleceram em homens, na segunda década de vida e região posterior de mandíbula. Os cistos foram mais prevalentes em relação aos tumores odontogênicos e o padrão de distribuição histológica de ambas as categorias foi similar ao encontrado na literatura, em que o cisto periapical e o ameloblastoma são as lesões predominantes.

Palavras-chave: Cisto odontogênico; Tumor odontogênico; Cisto periapical; Ameloblastoma; Perfil epidemiológico.

\section{Abstract}

Objective: this study aimed to establish the epidemiological profile of odontogenic lesions from the Department of Pathology and Forensic Medicine (DPML) of the Faculty of Medicine (FM) of the Federal University of Amazonas (UFAM), analyzing the most prevalent histological cyst types and odontogenic tumors, as well as their clinical features. Material and Methods: one hundred forty eight cases of odontogenic lesions were recovered between 1999 and 2014 from the DPML/FM/ UFAM and were analyzed for sex, age, anatomical site, and histological type. These data were submitted to descriptive analysis. Results: out of 148 patients with odontogenic lesions, slight predilection was observed for males $(54 \%, n=80)$, who were aged $20-30$ years $(34.1 \%, n=47)$, with the mandibular site being the most affected with $59.7 \%(n=77)$ of the cases. The histological type of lesions revealed $68.9 \%(n=102)$ cysts and $31.1 \%(n=46)$ odontogenic tumors. Of the cysts, the most prevalent histological type was the radicular cyst $(39.3 \%, n=40)$, and among tumors, the most common type was the ameloblastoma (45.6\%, $n=21)$. Conclusion: odontogenic lesions were prevalent in the posterior region of the mandible in males, aged 20-30 years. Cysts were more prevalent than odontogenic tumors and the pattern of histological distribution of both categories was similar to that found in the literature suggesting the predominance of the periapical cyst and ameloblastoma. Keywords: Odontogenic cyst; Odontogenic tumor; Radicular cyst; Ameloblastoma; Epidemiologic profile.

\section{Introdução}

A s lesões císticas e tumorais derivadas dos tecidos odontogênicos constituem grupos heterogêneos de lesões de grande relevância no campo da Patologia Oral e Maxilofacial. ${ }^{1,2}$ A prevalência dos tumores odontogênicos varia de acordo com a região anatômica, faixa etária, sexo, raça e localização geográfica. No Brasil, estudos relatam que a prevalência destas lesões varia entre 1,3 e 4,8\% de todas as lesões orais diagnosticadas. ${ }^{3-6}$ Os resultados de outras partes da América Latina, como México e Chile, são semelhantes. ${ }^{7.8}$ Estudos sobre a prevalência de cistos odontogênicos também foram realizados em vários países, incluindo o Brasil. No entanto, as informações nacionais são escassas. ${ }^{9}$

Vários estudos retrospectivos realizados na Ásia, África, Europa e América revelam que existem diferenças na frequ- ência relativa de vários tipos histológicos de tumores., ${ }^{310-12}$ Perfis epidemiológicos de cistos odontogênicos também têm sido relatados em diversos países do mundo, incluindo a Índia, ${ }^{1}$ Brasil,${ }^{9,13}$ Turquia, ${ }^{14}$ Espanha, ${ }^{15}$ entre outros. As variações na distribuição e frequência dos tipos de cisto, indicam uma diversidade epidemiológica entre os diferentes grupos populacionais. ${ }^{14}$

O presente estudo se propôs a revisar uma série de casos de lesões odontogênicas, ao longo de 15 anos, provenientes do Departamento de Patologia e Medicina Legal (DPML) da Faculdade de Medicina (FM) da Universidade Federal do Amazonas (UFAM) e assim estabelecer o perfil epidemiológico dessas lesões, analisando os tipos histológicos mais prevalentes de cistos e tumores odontogênicos, assim como suas características clinicopatológicas. Além disso, comparamos os dados obtidos neste estudo com aqueles relatados na literatura. 


\section{Material e Métodos}

Após aprovação pelo Comitê de Ética em Pesquisa da UFAM, foram recuperados 148 casos de lesões odontogênicas do livro de registro de lesões bucais do DPML/UFAM entre janeiro de 1999 a dezembro de 2014 (15 anos). Dados dos pacientes como sexo e idade foram coletados assim como os dados referentes às lesões propriamente ditas como localização anatômica e tipo de lesão de acordo com o diagnóstico histopatológico. Os laudos histológicos foram separados em cistos e tumores odontogênicos para posterior enquadramento na nova classificação da OMS de 2017 (Quadro 1), ${ }^{16,17}$ que foi colocada originalmente na OMS dentro do mesmo capítulo de tumores ósseos maxilofaciais. Os aspectos clínicos das lesões odontogênicas de maneira consolidada (cistos + tumores) foram discutidos e comparados com a literatura. Os casos diagnosticados como recidivas não foram considerados na amostra para evitar duplicidade de dados por se tratar do mesmo paciente.

Quadro 1. Classificação atualizada dos cistos e tumores odontogênicos (OMS, 2017)

\section{Carcinomas odontogênicos}

Carcinoma ameloblástico

$9270 / 3$

Carcinoma intraósseo primário, NOS

9270/3

Carcinoma odontogênico esclerosante

9270/3

Carcinoma odontogênico de células claras

$9341 / 3^{*}$

Carcinoma odontogênico de células fantasmas

9302/3*

Carcinosarcoma odontogênico

8980/3

Sarcoma odontogênico

9330/3

Tumores Odontogênicos Epiteliais Benignos

Ameloblastoma

9310/0

Ameloblastoma, tipo unicístico

9310/0

Ameloblastoma extraósseo/tipo Periférico

9310/0

Ameloblastoma metastatizante

9310/3

Tumor odontogênico escamoso

9312/0

Tumor odontogênico epitelial calcificante

9340/0

Tumor odontogênico adenomatoide

9300/0

Tumores Odontogênicos benignos mistos: epiteliais e mesenquimais

Fibroma ameloblástico

9330/0

Tumor odontogênico primordial

Odontoma

9280/0

Odontoma, tipo composto

$9281 / 0$

Odontoma, tipo complexo

9282/0

Tumor dentinogênico de células fantasmas

$9302 / 0$

Tumores Odontogênicos Mesenquimais Benignos

Fibroma odontogênico

$9321 / 0$

Mixoma odontogênico/Mixofibroma

9320/0

Cementoblastoma
Continuação quadro 1.

Fibroma cemento-ossificante

$9274 / 0$

Cistos odontogênicos de origem inflamatória

Cisto radicular

Cisto colateral inflamatório

Cistos de desenvolvimento odontogênicos e não odontogênicos

Cisto dentígero

Queratocisto odontogênico

Cisto periodontal lateral e cisto odontogênico

botrióide

Cisto gengival

Cisto odontogênico glandular

Cisto odontogênico calcificante

$9301 / 0$

Cisto odontogênico ortoqueratinizado

Cisto do ducto nasopalatino

Os códigos da morfologia são oriundos da Classificação Internacional de Doenças Oncológicas (ICD-O). O comportamente é codificado como: /0 para tumores benignos; /1 para inespecíficos, intermediários ou de comportamento desconhecido; / 2 para Carcinoma in situ ou Neoplasia Intraepitelial Grau III; /3 Para tumores malignos. A classificação foi modificada da classificação anterior da OMS, considerando as mudanças das lesões de acordo com o entendimento dos autores. ${ }^{*}$ Esses novos códigos foram aprovados pelo Comitê da "IARC/OMS" para ICD-0.

Fonte: Traduzido de El-Naggar et al. (2017).

Os aspectos clínicos dos cistos e tumores odontogênicos de maneira isolada foram também apresentados, porém a ênfase foi dada nos dados relacionados aos subtipos histológicos de cada categoria.

\section{Resultados}

Quanto às características clínicas dos 148 pacientes com lesões odontogênicas encontrados no estudo, 54\% $(\mathrm{n}=80)$ dos pacientes eram do sexo masculino e $46 \%(n=68)$ do sexo feminino. A média de idade da população estudada consistiu em 29,8 anos, acometendo pacientes no intervalo da faixa etária de 6 a 71 anos, com pico de prevalência na segunda e terceira décadas de vida, correspondendo a $34,1 \%$ $(\mathrm{n}=47)$ e $30,4 \%(\mathrm{n}=42)$, respectivamente. Em relação à localização anatômica, 40,3\% $(\mathrm{n}=52)$ dessas lesões ocorreram em maxila (sobretudo na região anterior) e 59,7\% $(\mathrm{n}=77)$ ocorreram em mandíbula (sobretudo na região posterior) (Tabela 1). 
Tabela 1. Distribuição dos pacientes acometidos por lesões odontogênicas de acordo com o sexo, idade e localização anatômica das lesões

\begin{tabular}{|c|c|c|c|}
\hline & $\begin{array}{l}\text { Lesões Odontogênicas } \\
100 \%(n=148)\end{array}$ & $\begin{array}{c}\text { Cistos } \\
68.9 \%(n=102)\end{array}$ & $\begin{array}{c}\text { Tumores } \\
31.1 \%(n=46)\end{array}$ \\
\hline \multicolumn{4}{|l|}{ Sexo } \\
\hline Masculino & $54 \%(n=80)$ & $54,9 \%(n=56)$ & $52,2 \%(n=24)$ \\
\hline Feminino & $46 \%(n=68)$ & $45,1 \%(n=46)$ & $47,8 \%(n=22)$ \\
\hline Total & $100 \%(n=148)$ & $100 \%(n=102)$ & $100 \%(n=46)$ \\
\hline \multicolumn{4}{|l|}{ Idade } \\
\hline $00-10$ & $4,4 \%(n=6)$ & $3,2 \%(n=3)$ & $7,2 \%(n=3)$ \\
\hline $11-20$ & $34,1 \%(n=47)$ & $30,3 \%(n=29)$ & $42,8 \%(n=18)$ \\
\hline $21-30$ & $30,4 \%(n=42)$ & $31,2 \%(n=30)$ & $28,5 \%(n=12)$ \\
\hline $31-40$ & $12,3 \%(n=17)$ & $13,5 \%(n=13)$ & $9,5 \%(n=4)$ \\
\hline $41-50$ & $6,5 \%(n=9)$ & $7,3 \%(n=7)$ & $4,8 \%(n=2)$ \\
\hline $51-60$ & $5,8 \%(n=8)$ & $6,3 \%(n=6)$ & $4,8 \%(n=2)$ \\
\hline$>60$ & $6,5 \%(n=9)$ & $8,3 \%(n=8)$ & $2,4 \%(n=1)$ \\
\hline Total & $100 \%(n=138 *)$ & $100 \%\left(n=96^{*}\right)$ & $100 \%(n=42 *)$ \\
\hline \multicolumn{4}{|c|}{ Localização Anatômica } \\
\hline Maxila (A) & $25,6 \%(n=33)$ & $22,2 \%(n=20)$ & $33,4 \%(n=13)$ \\
\hline Maxila (P) & $14,7 \%(n=19)$ & $20 \%(n=18)$ & $2,5 \%(n=1)$ \\
\hline Mandíbula (A) & $8,5 \%(n=11)$ & $6,7 \%(n=6)$ & $12,8 \%(n=5)$ \\
\hline Mandíbula (P) & $51,2 \%(n=66)$ & $51,1 \%(n=46)$ & $51,3 \%(n=20)$ \\
\hline Total & $100 \%(n=129 *)$ & $100 \%(n=90 *)$ & $100 \%\left(n=39^{*}\right)$ \\
\hline
\end{tabular}

*Dados incompletos. (A): Anterior; (P): Posterior

Em relação ao tipo de lesão odontogênica, observou-se que 68,9\% $(n=102)$ dos casos foram de cistos e $31,1 \%(n=46)$ de tumores odontogênicos. Os dados clínicos referentes aos cistos e tumores odontogênicos de maneira isolada também podem ser observados na tabela 1 .

Dentre os cistos odontogênicos, os subtipos mais prevalentes foram os de origem inflamatória com 55\% ( $\mathrm{n}=56$ ) do total, sendo o cisto periapical (ou radicular) o tipo histológico mais frequente correspondendo a 39,3\% $(\mathrm{n}=40)$, seguido pelo cisto dentígero (cisto de desenvolvimento) com 25,5\% $(\mathrm{n}=26)$ (Tabela 2).

Tabela 2. Distribuição dos subtipos de cistos odontogênicos encontrados de acordo com a origem

\begin{tabular}{|c|c|c|c|c|c|}
\hline Lesão & Origem & Tipo de cisto & Frequência (n) & Porcentagem (\%) & Total n (\%) \\
\hline \multirow{9}{*}{ Cisto } & \multirow{3}{*}{ Inflamatório } & Periapical & 40 & $39,3 \%$ & \multirow{3}{*}{$56(55,0 \%)$} \\
\hline & & Residual & 10 & $9,9 \%$ & \\
\hline & & Paradentário & 6 & $5,8 \%$ & \\
\hline & \multirow{4}{*}{ Desenvolvimento } & Dentígero & 26 & $25,5 \%$ & \multirow{4}{*}{$44(43,1 \%)$} \\
\hline & & Queratocisto & 14 & $13,8 \%$ & \\
\hline & & $\begin{array}{c}\text { Epitelial } \\
\text { Calcificante }\end{array}$ & 3 & $2,9 \%$ & \\
\hline & & Periodontal Lateral & 1 & $0,9 \%$ & \\
\hline & Sem especificação & Não especificado & 2 & $1,9 \%$ & $2(1,9 \%)$ \\
\hline & \multicolumn{2}{|c|}{ Total } & 102 & $100 \%$ & $102(100 \%)$ \\
\hline
\end{tabular}

Feita a análise dos 46 casos com diagnósticos de tumores odontogênicos, constatou-se que $54,3 \%(\mathrm{n}=25)$ destes possuem origem no epitélio odontogênico, $41,3 \%(\mathrm{n}=19)$ são de origem mista e os outros 4,4\% $(\mathrm{n}=2)$ dos casos tiveram origem no mesênquima (ectomesênquima) odontogênico. O tipo histológico mais frequente foi o ameloblastoma $(45,6 \%, \mathrm{n}=21)$, seguido pelo odontoma $(41,3 \%, \mathrm{n}=19)$ e tumor odontogênico adenomatoide $(8,7 \%, \mathrm{n}=4)$ (Tabela 3$)$. 
Tabela 3. Distribuição dos subtipos de tumores odontogênicos encontrados de acordo com a origem

\begin{tabular}{|c|c|c|c|c|c|}
\hline Lesão & Origem & Tipo de cisto & Frequência (n) & Porcentagem (\%) & Total n (\%) \\
\hline \multirow{8}{*}{ Tumor } & \multirow{2}{*}{ Epitelial } & Ameloblastoma & 21 & $45,6 \%$ & \multirow{2}{*}{$25(54,3 \%)$} \\
\hline & & Residual & 4 & $8,7 \%$ & \\
\hline & \multirow{3}{*}{ Misto } & Odontoma composto & 8 & $17,4 \%$ & \multirow{3}{*}{$19(41,3 \%)$} \\
\hline & & Odontoma complexo & 3 & $6,5 \%$ & \\
\hline & & Odontoma* & 8 & $17,4 \%$ & \\
\hline & \multirow{2}{*}{$\begin{array}{c}\text { Mesenquimal } \\
\text { (Ectomesenquimal) }\end{array}$} & Fibroma odontogênico & 1 & $2,2 \%$ & \multirow{2}{*}{$2(4,4 \%)$} \\
\hline & & Mixoma & 1 & $2,2 \%$ & \\
\hline & \multicolumn{2}{|c|}{ Total } & 46 & $100 \%$ & $46(100 \%)$ \\
\hline
\end{tabular}

TO= Tumor Odontogênico. *sem especificação

Foi encontrado apenas um paciente portador da Síndrome de Gorlin-Goltz, apresentando quatro lesões diagnosticadas como queratocistos odontogênicos.

\section{Discussão}

Estudos sobre frequência e características clínicas das lesões odontogênicas são importantes pois permitem saber mais precisamente a ocorrência destas lesões nas diversas populações, que por sua vez ajudam a identificar os grupos de risco e possíveis fatores associados ao seu desenvolvimento, bem como para desenvolver diagnósticos diferenciais mais precisos. ${ }^{5,15}$ Apesar do grande número de estudos sobre cistos odontogênicos e tumores odontogênicos na literatura, informações sobre o perfil epidemiológico destas lesões em diferentes populações é escasso, especialmente no caso da América Latina. Em geral, há menos relatos sobre a prevalência dos cistos odontogênicos do que sobre a dos tumores, apesar de os primeiros serem lesões muito mais prevalentes na população. ${ }^{6,18,19}$

A maioria das informações sobre a prevalência de cistos e tumores odontogênicos advém de serviços de diagnóstico e esses serviços representam uma fonte confiável de informações sobre a frequência relativa e características clínico-patológicas dessas lesões. ${ }^{19-21}$

Atualmente ainda não existem trabalhos epidemiológicos de lesões odontogênicas que utilizem a nova classificação da OMS, portanto, os artigos selecionados para a discussão e comparação dos resultados encontrados são, em sua maioria, baseados na classificação de 2005 de cistos e tumores odontogênicos da OMS onde os tumores odontogênicos queratocísticos e os tumores císticos calcificantes eram classificados como tumores. Vale, no entanto, ressaltar que nosso estudo baseia-se na classificação de 2017 da OMS, em que, por exemplo, as lesões (anteriormente tumorais) denominadas de tumor odontogênico queratocístico e tumor odontogênico cístico calcificante foram enquadradas novamente na categoria dos cistos odontogênicos, como queratocisto odontogênico e cisto odontogênico calcificante, respectiva- mente. ${ }^{16,17}$ Portanto, por ser uma classificação ainda muito recente, há dificuldade em se encontrar estudos semelhantes baseados na classificação mais atual que possam servir de comparativo.

No presente estudo, as lesões odontogênicas, incluindo cistos e tumores, foram semelhantes em ambos os sexos, sendo levemente prevalentes em pacientes do sexo masculino, estando de acordo com alguns estudos. ${ }^{20,22}$ Contudo, difere da maioria dos outros estudos que envolvem cistos e tumores odontogênicos, onde essas lesões foram mais prevalentes em pacientes do sexo feminino. ${ }^{18,23-27}$

Com relação à faixa etária, as lesões odontogênicas em nosso estudo apresentaram maior pico de incidência na segunda e terceira décadas de vida. Resultado este semelhante ao estudo de Borges et al. ${ }^{23}$ o qual utilizou uma grande casuística de lesões odontogênicas relatada no Brasil, mostrando prevalência dessas lesões principalmente na terceira década. No entanto, dados relativos à faixa etária são variados na literatura, possivelmente em função da casuística diversificada e tempo de coleta de dados nos mais diversos estudos, sejam nacionais ou internacionais. ${ }^{18,20,25,27}$

Quanto à localização anatômica, em nosso estudo a mandíbula, principalmente a região posterior, foi mais acometida do que a maxila pelas lesões odontogênicas, estando de acordo com outros inúmeros estudos, ${ }^{20,23,26}$ dentre eles, um estudo conduzido no Brasil por Borges et al..$^{23}$ Em contrapartida, há relatos da predominância dessas lesões em maxila, ${ }^{1,18}$ sendo um deles de um estudo indiano por um período de 20 anos.

Quanto ao tipo de lesão odontogênica, o presente estudo mostrou que os cistos odontogênicos ocorreram com maior frequência que tumores, estando, portanto, em plena concordância com a maioria dos estudos reportados na literatura. ${ }^{1,18,23,25}$ Isto pode ser devido ao fato de que os tumores odontogênicos por, na maioria dos casos, permanecerem assintomáticos durante o curso do processo de doença, só serem notados, em muitos casos, quando atingem grandes dimensões. ${ }^{4}$ Os cistos odontogênicos estão entre as lesões 
mais comuns que afetam os maxilares e muitos destes cistos compartilham características clínicas e radiográficas semelhantes, ${ }^{13}$ levando a necessidade de que o diagnóstico seja baseado em um exame rigoroso das características clínicas, radiológicas e histopatológicas. Em contrapartida, Pontes et al., ${ }^{24}$ por meio de um estudo epidemiológico de lesões odontogênicas tratadas sob anestesia geral, encontraram maior prevalêcia de tumores odontogênicos em relação aos cistos.

De maneira geral, em nossa revisão da literatura sobre o padrão de distribuição de lesões odontogênicas, verificamos que nossos resultados foram relativamente similares aos encontrados na literatura, sendo os cistos odontogênicos mais incidentes que os tumores odontogênicos como mostrado na tabela $4 .^{1,18,20,22-27}$ ra. ${ }^{1,9,13,18,20,29}$ Esta variação na incidência de cistos residuais pode ser devido à diferença na prevalência de cistos inflamatórios em várias populações e os protocolos de tratamento seguidos pelos cirurgiões-dentistas gerais no tratamento dessas lesões. ${ }^{9}$

O cisto paradentário é de origem inflamatória e acomete principalmente terceiros molares inferiores semi-inclusos, embora também haja relatos de casos associados a outros elementos dentários como o primeiro molar superior e o segundo pré-molar superior. ${ }^{15}$ Esses cistos desenvolvem-se próximo à margem cervical da porção lateral da raiz como consequência da inflamação da bolsa periodontal. Neste estudo, tal cisto foi pouco encontrado, havendo também inúmeras variações quanto a sua incidência na literatura,

Tabela 4. Distribuição de estudos epidemiológicos retrospectivos referentes a lesões odontogênicas

\begin{tabular}{|c|c|c|c|c|c|c|}
\hline Autores & Casuística & $\begin{array}{c}\text { Tempo da } \\
\text { pesquisa }\end{array}$ & $\begin{array}{c}\text { Sexo } \\
\text { Prevalente }\end{array}$ & $\begin{array}{c}\text { Faixa etária } \\
\text { prevalente }\end{array}$ & $\begin{array}{c}\text { Localização } \\
\text { prevalente }\end{array}$ & $\begin{array}{c}\text { Tipo de lesão } \\
\text { prevalente }\end{array}$ \\
\hline Pereira et al. ${ }^{18}$ (2010) & 43 & 10 anos & Feminino & $3 a$ e $\geq 60$ décadas & Maxila & Cisto \\
\hline Pontes et al. ${ }^{24}$ (2012) & 58 & 4 anos & Feminino & $\mathrm{NI}$ & Mandíbula & Tumor \\
\hline Borges et al. ${ }^{23}$ (2012) & 461 & 10 anos & Feminino & 2a-4a décadas & Mandíbula & Cisto \\
\hline Martins $^{27}$ (2012) & 86 & 3 anos & Feminino & $5 a$ década & $\mathrm{NI}$ & Cisto \\
\hline Al-Rawi et al. ${ }^{26}$ (2013) & 143 & 21 anos & Feminino & 2a-4a décadas & Mandíbula & Cisto \\
\hline Baghaei et al. ${ }^{25}$ (2014) & 81 & 13 anos & Masculino & 2a-4a décadas & Maxila & Cisto \\
\hline Ramachandra et al..$^{20}(2014)$ & 204 & 7 anos & Masculino & $\mathrm{NI}$ & Mandíbula & Cisto \\
\hline Niranjan \& Shaikh¹ (2014) & 763 & 20 anos & Masculino & $\mathrm{NI}$ & Maxila & Cisto \\
\hline Jaeger et al. ${ }^{22}$ (2017) & 5912 & 7 anos & Masculino & 1a a $5 a$ décadas & Mandíbula & Cisto \\
\hline Presente Trabalho (2017) & 148 & 15 anos & Masculino & 2a e 3a décadas & Mandíbula & Cisto \\
\hline
\end{tabular}

$\mathrm{NI}=$ não informado

Em relação aos cistos odontogênicos, nossos resultados mostraram que a maioria foi do tipo inflamatório estando de acordo com o percentual situado entre $61,4 \%-82,5 \%$ de prevalência como encontrado em outros estudos envolvendo populações da Índia, ${ }^{1}$ do Chile ${ }^{19}$ e do Brasil. ${ }^{13}$ Vale ressaltar que nesse estudo brasileiro, conduzido por Souza et al., ${ }^{13}$ os cistos odontogênicos foram analisados por um período de 38 anos

No presente estudo, dentre os cistos inflamatórios, o cisto periapical foi o mais prevalente, semelhante ao encontrado em vários estudos. ${ }^{1,9,19,28}$ Dentre os cistos de desenvolvimento, o cisto dentígero foi o mais comum, semelhante a diversos relatos. ${ }^{9,13,21,28,29}$ Em seguida, encontramos em nosso estudo, o segundo cisto de desenvolvimento mais frequente como sendo o queratocisto odontogênico que, de acordo com a nova classificação de $2017,{ }^{16,17}$ voltou para a categoria de cistos odontogênicos.

Cistos residuais, cistos paradentários, cisto odontogênico calcificante e cistos peridontais laterais foram os menos prevalentes em nosso estudo. Em contrapartida, uma variada prevalência do cisto residual já foi relatada na literatu- sobretudo em função das casuísticas e tempo de coleta dos dados. ${ }^{9,15,21,29}$

Os tumores odontogênicos são considerados lesões infrequentes, porém devem ser considerados durante o diagnóstico diferencial de lesões que envolvam os ossos maxilares. ${ }^{30}$ A literatura sobre a frequência relativa dos tumores odontogênicos no Brasil é escassa, em especial no Amazonas. No presente estudo, 46 casos foram diagnosticados em um período de 15 anos no Departamento de Patologia da UFAM constituindo $31,1 \%$ de todos os casos analisados.

No presente estudo, o ameloblastoma foi o tumor odontogênico prevalente nos ossos gnáticos, em concordância com diversos relatos da literatura. ${ }^{1,11,23,31}$ No entanto, outros relatos mostraram o odontoma como o tumor mais prevalente. ${ }^{22,26,32}$

Estudos relevantes e com grandes casuísticas como o de Avelar et al. ${ }^{4} \mathrm{e}$ da-Costa et al. ${ }^{5}$ mostraram maior prevalência para o tumor odontogênico queratocístico. Porém, vale relembrar que atualmente essa lesão é chamada de queratocisto odontogênico e migrou para a migrou para a categoria de cistos odontogênicos na classificação de 2017 da OMS $S^{16,17}$ 
cujo provavel impacto no perfil epidemiológico será no sentido de diminuir ainda mais o percentual de tumores em relação ao dos cistos odontogênicos.

O odontoma foi o segundo tumor mais prevalente em nosso estudo, sendo também o segundo mais comum em outros estudos. ${ }^{6,23,31}$ Em nosso estudo, o subtipo composto mais prevalente, divergindo de alguns estudos, ${ }^{8,31}$ onde o subtipo complexo foi o mais prevalente na amostra.

O tumor odontogênico adenomatoide apresentou baixa prevalência em nossa pesquisa. Outros estudos ${ }^{1,4,20,23}$ também monstraram uma baixa prevalência desses tumores. A ocorrência de mixomas odontogênicos e fibromas odontogênicos foram as menores. A baixa frequência dessas neoplasias na literatura, ${ }^{1,3,4,6,31}$ foi acompanhada pelos resultados encontrados no presente trabalho.

\section{Conclusão}

Como exposto, cistos e tumores odontogênicos apresentam diferenças quanto a sua prevalência, seja em uma análise individual de cada uma das lesões ou em conjunto. No presente estudo, as lesões odontogênicas prevaleceram em homens, na segunda década de vida e região posterior de mandíbula. Dados epidemiológicos em relação a esses aspectos têm sido variados na literatura, sobretudo em função da casuística diversificada e tempo de coleta dos dados, mas de maneira geral seguem um padrão de distribuição relativamente similar ao presente estudo. Os cistos odontogênicos foram mais prevalentes em relação aos tumores, fato observado na maioria dos estudos da literatura. O padrão de distribuição histológica mostrou que o cisto periapical e o ameloblastoma são as lesões odontogênicas predominantes, dentro das categorias de cistos e tumores odontogênicos, respectivamente, dado similar ao encontrado na maioria dos estudos relatados na literatura.

\section{Referências}

1. Niranjan KC, Shaikh Z. Clinicopathological correlation of odontogenic cysts and tumours in a South Indian population over a 20 -year period. Int J Dent Res. 2014;2(2):32-6.

2.Neville BW, Damm DD, Allen CM, Damm DD, Chi AC. Patologia Oral e Maxilofacial. Trad. 4. ed. Rio de Janeiro: Elsevier; 2016.

3. Ramos GO, Porto JC, Vieira DSC, Siqueira FM, Rivero ERC. Odontogenic tumors: a 14-year retrospective study in Santa Catarina, Brazil. Braz Oral Res. 2014;28(1):33-8.

4. Avelar RL, Antunes AA, Santos TDS, Andrade ESDS, Dourado E. Tumores odontogênicos: estudo clínico-patológico de 238 casos. Rev Bras Otorrinolaringol. 2008;74(5):668-73.

5. da-Costa DOP, Maurício AS, de-Faria PAS, da-Silva LE, Mosqueda-Taylor A, Lourenço SQC. Odontogenic tumors: A retrospective study of four Brazilian diagnostic pathology centers. Med Oral Patol Oral Cir Bucal. 2012;17(3):389-94.

6. Servato JP, Prieto-Oliveira PR, de-Faria AM, Loyola SV. Odontogenic tumours: 240 cases diagnosed over 31 years at a Brazilian university and a review of international literature. Int J Oral Maxillofac Surg. 2013;42(2):28893.

7. Mosqueda-Taylor A, Ledesma-Montes C, Caballero-Sandoval S, Portilla-Robertson J, Rivera LMRG, Meneses-García A. Odontogenic tumors in Mexico: a collaborative retrospective study of 349 cases. Oral Surg Oral Med Oral Pathol Oral Radiol Endod. 1997;84(6):672-5.

8. Ochsenius G, Ortega A, Godoy L, Peñafiel C, Escobar E. Odontogenic tumors in Chile: a study of 362 cases. J Oral Pathol Med. 2002;31(7):415-20. 9. Avelar RL, Antunes AA, Carvalho RWF, Bezerra PGCF, Neto PJO, Andrade ESS. Odontogenic cysts: a clinicopathological study of 507 cases. J Oral Sci. 2009;51(4):581-6.

10. Siriwardena BSMS, Tennakoon TMPB, Tilakaratne WM. Relative frequency of odontogenic tumors in Sri Lanka: Analysis of 1677 cases. Pathol Res Pract. 2012;208(4):225-30.

11. Lawal AO, Adisa AO, Olusanya AA. Odontogenic tumours: A review of 266 cases. J Clin Exp Dent. 2013;5:13-7.

12. Chrysomali E, Leventis M, Titsinides S, Kyriakopoulos V, Sklavounou A. Odontogenic tumors. J Craniomaxillofac Surg. 2013;24(5):1521-5.

13. Souza LB, Gordón-Núñez MA, Nonaka CW, Medeiros MC, Torres TF, Emiliano GBG. Odontogenic cysts: Demographic profile in a Brazilian population over a 38-year period. Med Oral Patol Oral Cir Bucal. 2010;15(4):58390.

14. Açikgöz A, Uzun-bulut E, Özden B, Gündüz K. Prevalence and distribution of odontogenic and nonodontogenic cysts in a Turkish Population. Med Oral Patol Oral Cir Bucal. 2012;17(1):108-15.

15. Nuñez-urrutia S, Figueiredo R, Gay-escoda C. Retrospective Clinicopathological study of 418 odontogenic cysts. Med Oral Patol Oral Cir Bucal.
2010;15(5):767-73.

16. El-Naggar AK, Chan JKC, Grandis JR, Takata T. World Health Organization Classification of tumors. Pathology and Genetics of Head and Neck tumors. 4th Lyon: IARC Press; 2017.

17. Wright JM, Vered M. Update from the 4th Edition of the World Health Organization Classification of Head and Neck Tumours: Odontogenic and Maxillofacial Bone Tumors. Head and Neck Pathol. 2017;11(1):68-77.

18. Pereira JV, Figueirêdo DU, Souza EA, Holmes TSV, Gomes DQC, Cavalcanti AL. Prevalência de cistos e tumores odontogênicos em pacientes atendidos na Fundação Assistencial da Paraíba: estudo retrospectivo. Arq Odontol. 2010;46(2):75-81.

19. Ochsenius G, Escobar E, Godoy L, Peñafil C. Odontogenic cysts: analysis of 2,944 cases in Chile. Med Oral Patol Oral Cir Bucal. 2007;12:85-9.

20. Ramachandra S, Shekar PC, Prasad S, Kumar KK, Reddy GS, Prakash $\mathrm{KL}$, et al. Prevalence of odontogenic cysts and tumors: A retrospective clinic-pathological study of 204 cases. SRM J Res Dent Sci. 2014;5(3):170-3.

21.Mosqueda-Taylor A, Irigoyen ME, Díaz MA, Torres MA. Odontogenic cysts: Analysis of 856 cases. Med Oral. 2002;7:89-96.

22. Jaeger F, Noronha MS, Silva MLV, Amaral MBF, Grossmann SDMC, Horta MCR, et al. Prevalence profile of odontogenic cysts and tumors on Brazilian sample after the reclassification of odontogenic keratocyst. J Craniomaxillofac Surg. 2017;45(2):267-70.

23. Borges LB, Fechine FV, Mota MRL, Sousa FB, Alves APNN. Odontogenic lesions of the jaw: a clinical-pathological study of 461 cases. Rev Gaúcha Odontol. 2012;60(1):71-8.

24. Pontes CGC, Neto AIT, Ribeiro ILH, Sarmento VA, Santos JN, Azevedo RA. Epidemiology of odontogenic cysts and tumors treated under general anesthesia in a philanthropic hospital in Salvador, Bahia. Rev Cir Traumatol Buco-Maxilo-Fac. 2012;12(1):93-100.

25. Baghaei F, Zargaram M, Najmi H, Moghimbeigi A. A clinicopathological study of odontogenic cysts and tumors in Hamadan, Iran. J Dent. 2014;15(4):167-72.

26. Al-Rawi NH, Awad M, Al-Zuebi IE, Hariri RA, Salah EW. Prevalence of odontogenic cysts and tumors among UAE population. J Orofac Sci. 2013;5(2):95-100.

27. Martins TH. Cistos e tumores odontogênicos: Estudo retrospectivo. 26 f. Trabalho de conclusão de curso (Monografia)- Universidade Estadual de Londrina, Londrina, 2012.

28. Prockt AP, Schebela CR, Maito FDM, Sant'Ana Filho M, Rados PV. Odontogenic Cysts: Analysis of 680 Cases in Brazil. Head and Neck Pathol. 2008;2:150-6.

29. Sharifian MJ, Maryam K. Odontogenic cysts: a retrospective study of 1227 cases in an Iranian population from 1987 to 2007. J Oral Sci. 2011;53(3):361-7. 30. Antunes AA, Silva JL, Silva PV, Antunes AP. Tumores odontogênicos: 
Análise de 128 casos. Rev Bras Cir Cabeça Pescoço. 2006;35(3):160-3.

31. Saghravanian N, Jafarzadeh H, Bashardoost N, Pahlavan, N, Shirinbak I.

Odontogenic tumors in an Iranian population: a 30 -year evaluation. J Oral
Sci. 2010;52(3):391-6.

32. Santos JN, Pinto LP, Figueredo CR, Souza LB. Odontogenic tumors: analysis of 127 cases. Pesqui Odontol Bras. 2001;15(4):308-13.

\section{Mini Currículo e Contribuição dos Autores}

1. Brendo Vinicius Rodrigues Louredo - graduando em Odontologia. Contribuição: execução do trabalho, levantamento bibliográfico e redação do manuscrito.

2. Camila Tatyanne Santos de Freitas - cirurgiã-dentista e residente em Patologia Oral e Maxilofacial. Contribuição: tabulação de dados, redação e revisão do manuscrito.

3. Jeconias Câmara - cirurgião-dentista e mestre. Contribuição: coorientação do trabalho e revisão do manuscrito.

4. Tatiana Nayara Libório Kimura - Cirurgiã-dentista e PhD. Contribuição: orientação do trabalho, revisão do manuscrito, aprovação da versão final e submissão à revista.

Recebido em: 21/03/2017 / Aprovado em: 18/05/2017

Autora Correspondente

Tatiana Nayara Libório-Kimura

E-mail: tliborio@ufam.edu.br 